\title{
Design The Contactless Charger AND CONTACTless DATA TRANSFER BETWEEN UNDERWATER ROBOT- SATELLITS AND UNDERWATER 6-LEGGED VEHICLE
}

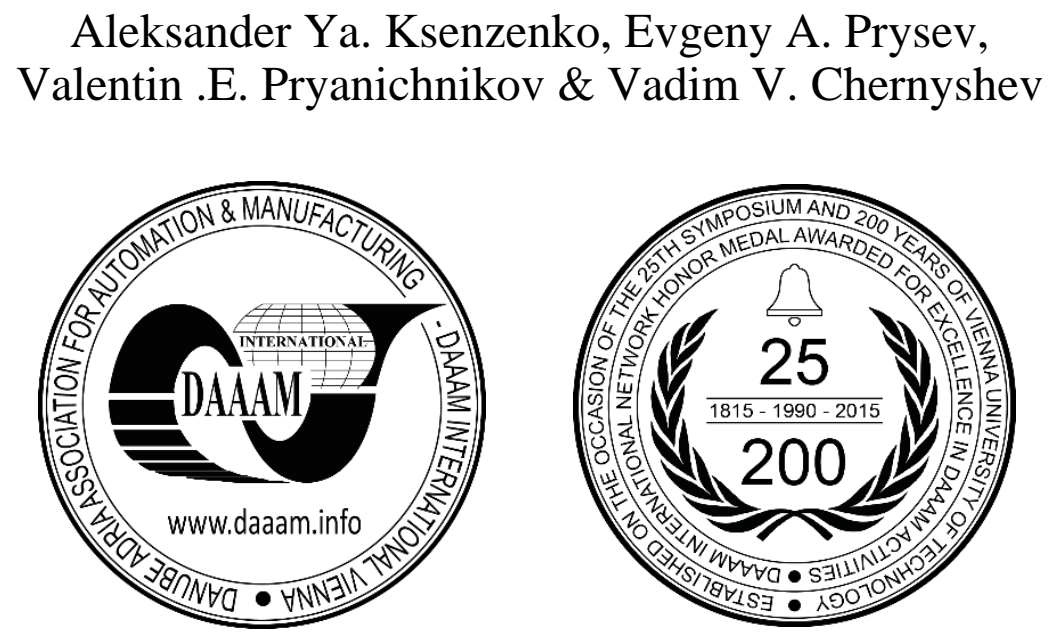

This Publication has to be referred as: Ksenzenko, A[lexander]; Prysev, E[vgeny]; Pryanichnikov, V[alentin] E[.] \& Chernyshev, V[adim] (2017). Design the Contactless Charger and Contactless Data Transfer between Underwater RobotSatellits and Underwater 6-Legged Vehicle, Proceedings of the 28th DAAAM International Symposium, pp.1197-1201, B. Katalinic (Ed.), Published by DAAAM International, ISBN 978-3-902734-11-2, ISSN 1726-9679, Vienna, Austria DOI: $10.2507 / 28$ th.daaam.proceedings. 166

\begin{abstract}
The work is devoted to prototyping of the 6-legged walking underwater vehicle as base station with contactless charger and contactless data transfer between itself and a number of autonomous underwater robot-sputniks. An architecture is proposed to build the robotic complex as interacting parts had shown the efficiency. The separation of slow-moving, but well passable on the bottom legged platform, connected by cable with a remote supervisory control station on a boat, and highly maneuverable, fully self-contained units - direct executors for underwater operations significantly extend the operational capabilities of the complex. This hardware build simplified the organization of the underwater operations due to of rapid charging of autonomous modules' batteries, directly in the area of the works, without their ascent to the surface, as well as the separation of the control channel into two parts: the cable and sonar lines. The work is executed at partial financial support of RFBR, projects 16-07-01264, 16-07-00811, 16-07-00935.
\end{abstract}

Keywords: underwater robotics; underwater contactless charger; underwater contactless data transfer; remote supervisory control

\section{Introduction}

Exploration of unmanned robotic devices in the study and practical use of spaces that are hidden under water becomes increasingly important because the problem of ensuring human security under water is avoided. The underwater robots constructed for this purpose can be conditionally divided into two groups: (1) fully autonomous underwater vehicles and (2) tethered devices connected by a cable to a control station that is located in a safe place (such as on the coast). Each of these groups has certain advantages and disadvantages. Despite significant advantages of autonomous underwater robots, their widespread use is hindered by the problem of uninterrupted power supply and the complexity of arranging a remote underwater control channel by the operator. Because of this, fully autonomous unmanned underwater vehicles turn out to be rather large, complex, and expensive in terms of their construction and operation. 
To avoid this contradiction, we propose a configuration of the robotic system consisting of three separate yet interacting devices. The first device is a limited mobile base station connected by a cable to the control station that is located, for example, on the coast. This cable ensures power supply for all underwater components of the system. The cable serves for the transfer of control commands by both the base station and autonomous modules through a hydroacoustic communication channel.

The second device is a group of fully autonomous underwater modules that are relatively small in size, have no external connecting cable, and provide increased maneuverability; they are responsible for the direct execution of underwater operations several dozens of meters away from the base station. The modules are to be controlled by the operator station through a communication channel consisting of two parts: by a cable extending from the control panel to the base station and through a short hydroacoustic communication channel extending to each autonomous module. In addition, each module is equipped with a device for contactless operational recharging of its batteries from the base station just in the workplace (without ascending).

The third device is a control station (with operators) located on the coast or on an accompanying boat. The operator controls the movement of the base station along the bottom into the workplace with the help of on-board video sensors through a wire communication channel. The hydroacoustic channel and the base station are used by the operator to conduct all the manipulations with the autonomous modules required for relevant actions in the workplace.

This architecture of hardware for the underwater system makes it possible to combine the operational advantages of fully autonomous robotic unmanned robots with the convenience in organizing a channel for their remote control.

The underwater robotic system consists of several complex interconnected functional systems. This study considers the most important of these systems that go through the stages of prototyping and testing. Further, we consider a device of 6-legged walking construct of the base station, a contactless system for recharging on-board batteries of autonomous modules directly in the workplace without the need for their ascent, and a system for exchanging large data amounts between the base station and autonomous modules through a high-speed radio channel in salty water.

\section{Composition and structure of the base station}

The base station is functionally an intermediate link between the actuator operating in extreme conditions and the operator situated in rather comfortable conditions at the central control station. In terms of construction, the base station is designed as a self-moving 6-legged walking device, with the following equipment to be installed in sealed boxes:

- two identical stepped modules (right and left boards);

- movers of each board;

- front and rear view television cameras;

- powerful LED searchlights with visible and infrared light;

- devices for mooring of autonomous modules with the contactless recharging system;

- hydroacoustic antennas for channels of control over autonomous modules;

- an ultrasonic beacon for orienting the autonomous modules under reduced visibility;

- an electronic compass outputting current information about the location azimuth.

Each board has movers each consisting of three cyclic pacing mechanisms with an adjustable trajectory of the reference point. The pacing mechanisms in the mover are linked kinematically and act in antiphase: two of them act in phase and the third acts in antiphase. The step size and height can be operationally changed from 80 to $120 \mathrm{~cm}$ and from 20 to $60 \mathrm{~cm}$, respectively. The electric drives of pacing mechanisms are made on asynchronous electric motors with a frequency regulation of speed and a total power of around $1500 \mathrm{~W}$. The motion speed of the base station along the flat bottom reaches $5 \mathrm{~km} / \mathrm{h}$ and depends basically on the bottom surface features and the visibility conditions of the route. Electricity and control signals arrive from the control station through a single-wire coaxial hoist cable; the length of this cable can reach $120 \mathrm{~m}$. The base station can be rotated driven by different speeds of mover drives of each board. The weight of the experimental operating model of the base station is around $170 \mathrm{~kg}$.

The movers of the left and right boards are made according to cyclic pacing mechanisms acting in antiphase. The cyclic movers make it possible to maintain the stability of pacing and do without a controlled adaptation system. It is known that the pacing machines with cyclic-type movers have simple kinematic drives and are simpler than their analogs with adaptive control of pacing. Unfortunately, the fact that the trajectory of reference points for cyclic pacing mechanisms is invariant does not allow the mover adaptation capabilities to be fully explored when the character of motion path profile varies. Therefore, the overcoming of obstacles makes allowance for controlling the step length and height as well as changing the position of reference points when turning to reduce the moment of resistance to rotation. The trajectory of reference points is adjusted by additionally introducing a special crank with a linear electric drive into the pacing mechanism; in this case, a single mechanism can adjust the trajectory of all three pacing mechanisms of its board. The control of the trajectory of reference points of the pacing mechanism is reduced to a discrete change in the angular position of the additional crank. This leads to a shift in the arm suspension point and a transformation of the base trajectory of marching motion into the trajectory of obstacle handling with increased step height and length [1-3]. 
The contactless recharging of the power of onboard batteries is conducted through a device based on the principle of electrodynamic induction. The radiating part of this device is installed inside the sealed casing of the base station and contains a generator of high-frequency oscillations with a power of around $95 \mathrm{~W}$ and a directional antenna. The generator is supplied with power from the base station network. High-frequency voltage is supplied to the radiating antenna that is located under the radio-transparent cover of the device. The hydro pump built into the mooring mechanism provides adequate positioning and holding of the mobile module in a required place during the whole period of contactless power transmission, which can last up to several hours.

Due to its own movers and video cameras, the base station can be moved by operator's commands to the workplace and fixed at this place. The base station has a hydroacoustic antenna to control the operation of autonomous modules. When the bottom is muddy, the visibility is reduced to less than a meter; in view of this, the base station should contain an ultrasonic beacon along with powerful searchlights.

\section{Composition and structure of the autonomous modules}

There should be at least two autonomous modules (highly maneuverable underwater robotic devices). The equipment of the module can be entirely placed in a sealed casing. An autonomous module must be equipped with:

- movers;

- on-board batteries;

- lamps and video cameras;

- a receiver of the system of contactless recharging of onboard batteries;

- a receiver/transmitter of the system of operational exchange of large data amount;

- a proper navigation system;

- a hydroacoustic system of communication through the base station with the central control station;

- an on-board computer;

- auxiliary actuators installed on the module for specific operations.

The actual efficiency of underwater operations by automated devices depends essentially on the maximum possible time of their continuous operation without ascending to the surface. Periodic interruptions are caused by the need to recharge the onboard battery of the power supply system as well as to promptly adjust and update the onboard computer software. To this end, one has to stop the operations, ascend the device to the water surface, unseal it, recharge or replace the batteries, reseal the device, execute control operations, submerge, search, move and position the device in the workplace. With an increase in the depth of operations, the importance of increased length of continuous operation grows substantially.

The method of power recharging of on-board batteries of autonomous modules makes it possible to operate directly in the workplace and avoid ascending to the surface. To solve this problem taking into account the large depth and salty water medium, the autonomous module is equipped with a receiver of the contactless energy transfer system. The autonomous module uses its navigation system, hydroacoustic beacons, and video cameras to approach the radiating antenna of the base station. This can be conducted both by the operator situated at the control post and in a fully automatic mode using a special-purpose program. Since the grouping of autonomous modules interacts with the base station, the operations are not interrupted during the charging of the moored autonomous module: another module with a previously charged battery is activated.

The receiver of the autonomous module is located behind the radio-transparent wall of the casing. The electromagnetic field radiated by the transmitting antenna of the base station is captured by the receiving antenna of the autonomous module and converted into electric current. A special-purpose device converts this current into a normalized current of battery charge. This device provides an optimal mode of battery charging and protects it from recharging. The degree of readiness of the battery for operation is controlled by the on-board computer and can be visualized on the control station display $[4,5]$.

The autonomous modules should be controlled with the help of a navigation system with a hydroacoustic duplex communication channel. Being relatively narrow-band, this channel cannot provide high quality television imagery; nevertheless, the quality is sufficient to meet the technical requirements of remote control. When using the range of ultrasonic frequencies of around several hundred kilohertz for the transmission of a good quality video signal and the reception of control commands, the acceptable distance of the mobile module to the base station is approximately 20-40 $\mathrm{m}$. Thus, the control station operator can remotely perform underwater operations with the help of a manoeuvrable mobile module, using a high-quality cable communication channel to the base station and a short hydroacoustic section of the communication channel to the mobile module.

Autonomous modules can collect information from underwater objects of different origin and purpose. Normally, this is information is rather extensive: telemetry for a long period of observations, high-quality photo and video materials, etc. To this end, the autonomous module and the base station must be equipped with a system that can exchange large data amounts through a broadband radio channel capable of operating in a salty water medium. With a broadband radio channel at a frequency of $2.4 \mathrm{GHz}$, the attenuation in salty water is around $85 \mathrm{~dB}$ per meter. 
Despite this fact, the signal at a distance of several centimetres was sufficiently powerful to transfer data efficiently from the module to the base, in the mode of base station docking during battery recharge (Fig. 1). This communication channel can also be used to remotely update the computing software at facilities of the autonomous module (Fig. 2). The exchange channel of large data arrays organized in this way is highly secure against unauthorized reading of transmitted data.

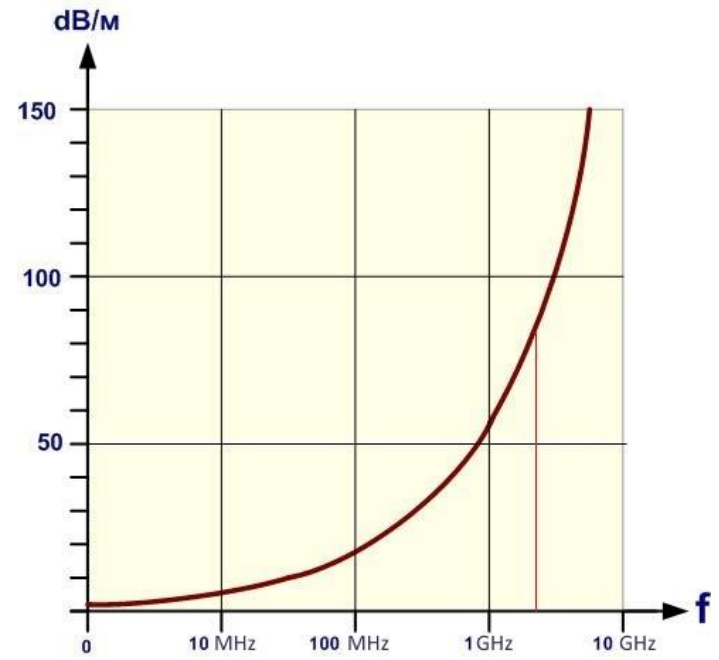

Fig. 1. Typical dependence of seawater radio transparency on frequency

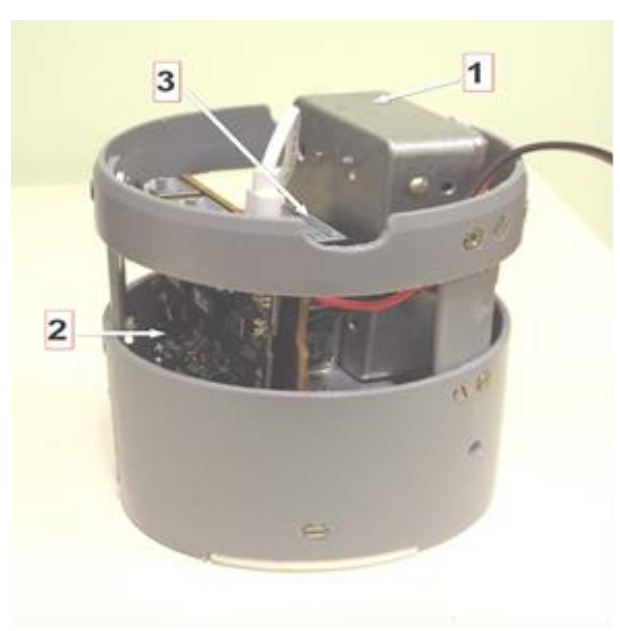

Fig. 2. Fragment of the radiating device for contactless charging and the receiver/transmitter system of the radio channel of exchange of large data amounts.

(1) Generator of high-frequency voltage for the system of contactless charging, (2) Board of the receiver/ transmitter system of the radio channel of exchange of large data amounts,

(3) Auxiliary voltage inverter of the power receiver/ transmitter.

\section{The principles of control between elements of robotic system.}

The software of the robotic system should serve the mobile module control system and the base station control system. This is done by on-board computers that provide supervisory control, motion programming, and telemetry analysis. The possibility of motion programming without interrupting the current operations is ensured by using a technology that uses of an interpreted dynamic language for the upper level of control.

This allows one to promptly edit the on-board computer programs and organize exchange of messages between software components with the help of the actor model; this makes it possible to partially solve the problem of recovery after a network failure in data exchange and accumulate the results of telemetry in a non-relational database. A similar technology was successfully developed on Amur ground-based mobile robots.

\section{Full-scale testing.}

Tests of pacing characteristics of the current prototype of the pacing base station were conducted under different bottom conditions at a depth of up to $20 \mathrm{~m}$ in the Kandalaksha Gulf of the White Sea (Fig. 3). Different modes of straightline motion and rotations were considered with various foot designs.

The design tests revealed that the pacing machine in terms of passing ability along the sea bottom is much superior to wheel and caterpillar movers. The advantages of the pacing mover manifest themselves most conclusively when moving along a complex rocky bottom.

The tests indicated that the system of contactless recharging of on-board batteries is efficient, with the coefficient of efficiency (taking into account the inaccuracy of positioning of docking nodes and water salinity) being in the range of 30 to $45 \%$ (Fig. 4). 


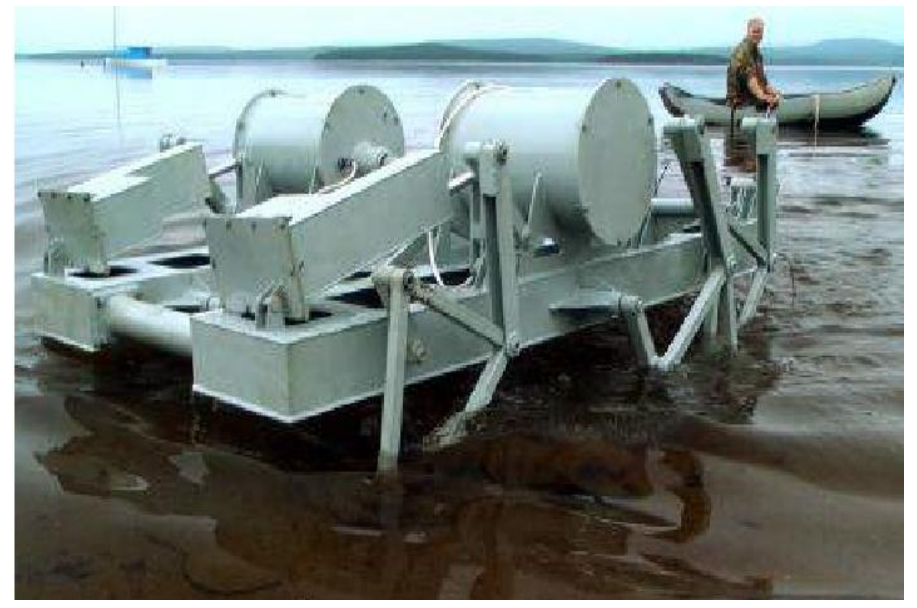

Fig. 3. Motion of the pacing machine of the base system along the bottom at a small depth.

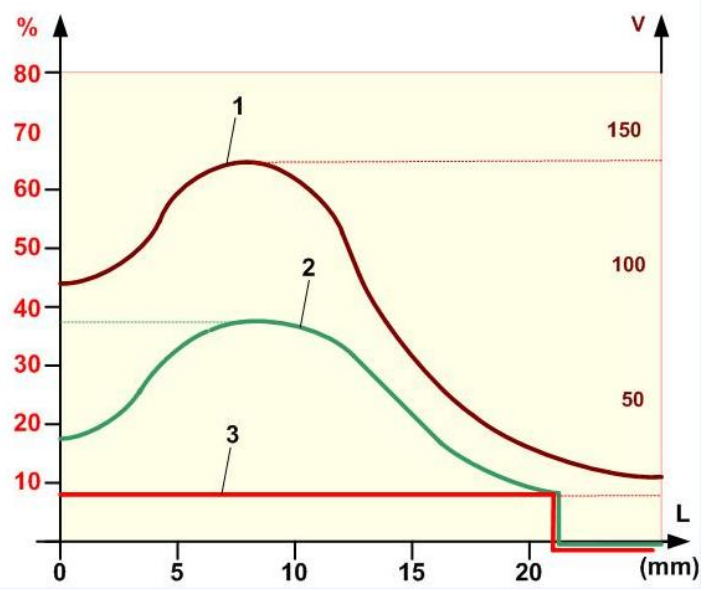

Fig. 4. Efficiency of contactless power transfer for underwater devices.

(1) Voltage at the output of receiving antenna,

(2) Coefficient of efficiency,

(3) Output voltage of charging device.

\section{Conclusion.}

The hardware construction principles for robotic systems developed here by combining the low-maneuverable (but highly passable along the bottom) tethered base station with several highly maneuverable autonomous mobile modules as well as by using a contactless method of battery charging make it possible to construct vehicle with qualitatively new operational capabilities (specifically, when operating on complex and almost inaccessible bottom reliefs and in submerged constructs).

\section{References.}

[1] Chernyshev V.V., Arykantsev A.E., Gavrilov, A., Control of the motion of underwater pacing devices moving along the bottom, Inv. YuFU, Technical Sciences, Proc. of All-Russian Conf. "Prospective Systems and Control Problems", 2016, no. 1, pp. 141-155.

[2] Chernyshev V.V., Simulation of the dynamics of pacing machine with cycling movers as a system of rigid bodies with elastic-dissipative coupling, Izv. VolgGTU: Ser. Important problems of control, computers, and informatics in technical systems, 2010, no. 11, pp. 32-35.

[3] Chernyshev V., Arykantsev V., Kalinin Y., Gavrilov A., Sharanov N., Development of the walking mover for underwater walking vehicle, Proccedings of the 26th DAAAM International Symposiumon, 2016, Katalinic B. (Ed.), Published by DAAAM International, Vienna, Austria, pp. 1143-1148.

[4] Bielich T., Kirsanov K.B., Ksenzenko A.Ya., Pryanichnikov V.E., Increasing the time of continuous operation of the underwater inspectoral robotic system, Information-measuring and control systems, Moscow: Radiotekhnika, 2015, vol. 13, no. 7, pp. 57-65.

[5] Pryanichnikov V.E., Ksenzenko A.Ya., Kuvshinov S.V., Poduraev Yu.V., Prysev E.A., Khelemendik R.V., Eprikov S.R. Intelligent rodotronics: hardware-software complexes of rodotariums. 25th DAAAM International Symposium on Intelligent Manufacturing and Automation 2014, Procedia Engineering, 2015, vol. 69, no. 1, pp. 35-40. DAAAM International, Vienna, Austria. 